\title{
Parameterizations and Lagrange Cubics for Fitting Multidimensional Data
}

Ryszard Kozera $^{1,2,3(\bowtie)}$, Lyle Noakes ${ }^{2}$, and Magdalena Wilkołazka ${ }^{3(\bowtie)}$

1 Institute of Information Technology, Warsaw University of Life Sciences - SGGW, Ul. Nowoursynowska 157, 02-776 Warsaw, Poland ryszard.kozera@sggw.edu.pl, ryszard.kozera@gmail.com

2 Faculty of Engineering and Mathematical Sciences, The University of Western Australia, 35 Stirling Highway, Crawley, Perth, WA 6009, Australia lyle.noakes@uwa.edu.au

3 Faculty of Natural and Health Sciences,

The John Paul II Catholic University of Lublin, Ul. Konstantynów 1H, 20-708 Lublin, Poland

magda.wilkolazka@gmail.com, magda8310@kul.lublin.pl

\begin{abstract}
This paper discusses the issue of interpolating data points in arbitrary Euclidean space with the aid of Lagrange cubics $\hat{\gamma}^{L}$ and exponential parameterization. The latter is commonly used to either fit the so-called reduced data $Q_{m}=\left\{q_{i}\right\}_{i=0}^{m}$ for which the associated exact interpolation knots remain unknown or to model the trajectory of the curve $\gamma$ passing through $Q_{m}$. The exponential parameterization governed by a single parameter $\lambda \in[0,1]$ replaces such discrete set of unavailable knots $\left\{t_{i}\right\}_{i=0}^{m}\left(t_{i} \in I\right.$ - an internal clock) with some new values $\left\{\hat{t}_{i}\right\}_{i=0}^{m}$ $\left(\hat{t}_{i} \in \hat{I}\right.$ - an external clock). In order to compare $\gamma$ with $\hat{\gamma}^{L}$ the selection of some $\phi: I \rightarrow \hat{I}$ should be predetermined. For some applications and theoretical considerations the function $\phi: I \rightarrow \hat{I}$ needs to form an injective mapping (e.g. in length estimation of $\gamma$ with any $\hat{\gamma}$ fitting $Q_{m}$ ). We formulate and prove two sufficient conditions yielding $\phi$ as injective for given $Q_{m}$ and analyze their asymptotic character which forms an important question for $Q_{m}$ getting sufficiently dense. The algebraic conditions established herein are also geometrically visualized in $3 D$ plots with the aid of Mathematica. This work is supplemented with illustrative examples including numerical testing of the underpinning convergence rate in length estimation $d(\gamma)$ by $d(\hat{\gamma})$ (once $m \rightarrow \infty$ ). The reparameterization has potential ramifications in computer graphics and robot navigation for trajectory planning e.g. to construct a new curve $\tilde{\gamma}=\hat{\gamma} \circ \phi$ controlled by the appropriate choice of interpolation knots and of mapping $\phi$ (and/or possibly $Q_{m}$ ).
\end{abstract}

\section{Introduction}

Assume that $\gamma: I \rightarrow \mathbb{E}^{n}$ represents a smooth regular curve (i.e. $\dot{\gamma}(t) \neq \overrightarrow{0}$ ) of class $C^{k}$ (usually with $k=3,4$ ) defined over a compact interval $I=[0, T]$ (with 
$0<T<\infty)$. Suppose that $m+1$ interpolation points $\left\{q_{i}\right\}_{i=0}^{m}=\left\{\gamma\left(t_{i}\right)\right\}_{i=0}^{m}$ (forming the so-called reduced data $Q_{m}$ ) belong to an arbitrary Euclidean space $\mathbb{E}^{n}$. Here $\mathcal{T}=\left\{t_{i}\right\}_{i=0}^{m}$ is not given (here $t_{i}<t_{i+1}$ ). We introduce now (see e.g. $[1,7,12]$ or $[19]$ ) some preliminary notions (applicable for $m \rightarrow \infty$ ).

Definition 1.1. The interpolation knots $\mathcal{T}$ are admissible if:

$$
\lim _{m \rightarrow \infty} \delta_{m}=0, \text { where } \delta_{m}=\max _{1 \leq i \leq m}\left\{t_{i}-t_{i-1}: \quad i=1,2, \ldots, m\right\} .
$$

Definition 1.2. The interpolation knots $\mathcal{T}$ are more-or-less uniform if there exist constants $0<K_{l} \leq K_{u}$ such that:

$$
\left(K_{l} / m\right) \leq t_{i}-t_{i-1} \leq\left(K_{u} / m\right),
$$

for all $i=1,2, \ldots, m$ and any $m \in \mathbb{N}$. Alternatively, more-or-less uniformity amounts to the existence of some constant $0<\beta \leq 1$ such that $\beta \delta_{m} \leq t_{i}-t_{i-1} \leq$ $\delta_{m}$ for all $i=1,2, \ldots, m$ and arbitrary $m \in \mathbb{N}$. Lastly, the subfamily $\mathcal{T}_{\beta_{0}}$ of moreor-less uniform samplings represents a set of $\beta_{0}$-more-or-less uniform samplings if each of its representatives satisfies $\beta_{0} \leq \beta \leq 1$, for some $0<\beta_{0} \leq 1$ fixed.

Having selected the fitting scheme $\hat{\gamma}$ of $Q_{m}$ the unknown knots $\mathcal{T}$ for the interpolant $\hat{\gamma}$ must somehow be replaced by estimates $\hat{\mathcal{T}}=\left\{\hat{t}_{i}\right\}_{i=0}^{m}$ subject to $\hat{\gamma}\left(\hat{t}_{i}\right)=q_{i}$. We use here the so-called exponential parameterization (see e.g. [17]) which depends on a single parameter $\lambda \in[0,1]$ according to:

$$
\hat{t}_{0}=0 \text { and } \hat{t}_{i}=\hat{t}_{i-1}+\left\|q_{i}-q_{i-1}\right\|^{\lambda},
$$

for $i=1,2, \ldots, m$. It is also assumed here that $q_{i} \neq q_{i+1}$ so that the extra condition $\hat{t}_{i}<\hat{t}_{i+1}$ is preserved as stipulated generically while fitting reduced data $Q_{m}$. The case of $\lambda=0$ in (3) gives uniform knots $\hat{t}_{i}=i$. Evidently the latter does not reflect the geometry of $Q_{m}$. On the other hand, $\lambda=1$ yields the so-called cumulative chord parameterization which coincides with Euclidean distances between consecutive points $q_{i}$ and $q_{i+1}$ and as such it refers to the spread of $Q_{m}$. More information on the above topic and related issues can be found e.g. in $[3,5,16,17]$ or $[18]$.

The selection of the specific interpolant $\hat{\gamma}: \hat{I}=[0, \hat{T}] \rightarrow \mathbb{E}^{n}$ (with $\hat{T}=$ $\hat{t}_{m}$ ) together with some knots' estimates $\hat{\mathcal{T}} \approx \mathcal{T}$ raises an important question concerning the convergence rate (if any) in approximating $\gamma$ with $\hat{\gamma}$ (or its length) once $m \rightarrow \infty$. Recall first (see $[1,12]$ or $[19]$ ):

Definition 1.3. Consider a family $\left\{F_{\delta_{m}}, \delta_{m}>0\right\}$ of functions $F_{\delta_{m}}: I \rightarrow \mathbb{E}^{n}$. We say that $F_{\delta_{m}}$ is of order $O\left(\delta_{m}^{\alpha}\right)$ (denoted as $F_{\delta_{m}}=O\left(\delta_{m}^{\alpha}\right)$ ), if there is a constant $K>0$ such that, for some $\bar{\delta}>0$ the inequality $\left\|F_{\delta_{m}}(t)\right\|<K \delta_{m}^{\alpha}$ holds for all $\delta_{m} \in(0, \bar{\delta})$, uniformly over $I$.

For a given $\hat{\gamma}$ fitting dense data $Q_{m}$ based on $\hat{\mathcal{T}} \approx \mathcal{T}$ (and some a priori selected mapping $\phi: I \rightarrow \hat{I}$ ) the natural question arises about the distance measurement $\left\|F_{\delta_{m}}\right\|=\|\gamma-\hat{\gamma} \circ \phi\|$ tending to 0 (uniformly over $I$ ), while $m \rightarrow \infty$. 
Of course, by (1) proving $F_{\delta_{m}}=\gamma-\hat{\gamma} \circ \phi=O\left(\delta_{m}^{\alpha}\right)$ not only guarantees the latter but also establishes lower bound on convergence speed (if $\alpha>0$ ). The coefficient $\alpha>0$ appearing in Definition 1.3 is called the convergence rate in approximating $\gamma$ by $\hat{\gamma} \circ \phi$ uniformly over $[0, T]$. If additionally such $\alpha$ cannot be improved (once $\gamma$ and $\mathcal{T}$ are given) then $\alpha$ is sharp. The latter analogously extends to the length estimation (with $n=1$ ), for which the scalar expression $F_{\delta_{m}}=d(\gamma)-d(\hat{\gamma})=O\left(\delta_{m}^{\beta}\right)$ is to be considered.

For certain applications such as the analysis of the convergence rate in $d(\gamma)=$ $\int_{0}^{T}\|\dot{\gamma}(t)\| d t \approx d(\hat{\gamma})=\int_{0}^{\hat{T}}\left\|\hat{\gamma}^{\prime}(\hat{t})\right\| d \hat{t}$ (see e.g. [2,5] or [15]) the mapping $\phi(t)=\hat{t}$ should be a reparameterization of $I$ into $\hat{I}$ (i.e. $\dot{\phi}>0$ ). In other situations such as robot's and drone path planning the extra trajectory looping of $\hat{\gamma}$ is sometimes needed (e.g. for traction line posts' inspection while making circles by drone). Of course, in many other applications robot navigation requires trajectory planning with no loops whatsoever. In that context (as well as for length estimation) one of the conditions to exclude the local looping of $\hat{\gamma} \circ \phi$ is to require $\phi$ to be an injective function (see e.g. [13]).

From now on it is assumed that $\hat{\gamma}=\hat{\gamma}^{L}$ which represents a piecewiseLagrange cubic $\hat{\gamma}^{L}: \hat{I}=[0, \hat{T}] \rightarrow \mathbb{E}^{n}$ (see e.g. [1]). More precisely, the interpolant $\hat{\gamma}^{L}$ is defined as a track-sum of Lagrange cubics $\left\{\hat{\gamma}_{i=3 k}^{L}\right\}_{k=0}^{(m-3) / 3}$ with each $\hat{\gamma}_{i}^{L}: \hat{I}_{i}=\left[\hat{t}_{i}, \hat{t}_{i+3}\right] \rightarrow \mathbb{E}^{n}$ satisfying $q_{i+j}=\hat{\gamma}_{i}^{L}\left(t_{i+j}\right)$, for $j=0,1,2,3$. As already pointed out the unavailable knots $\mathcal{T}$ are estimated with $\hat{\mathcal{T}}$ governed by exponential parameterization (3). For simplicity we suppose that $m=3 k$, where $k \in\{1,2,3, \ldots\}$. In a similar fashion, one selects here $\phi=\psi^{L}$ defined as a tracksum of Lagrange cubics $\left\{\psi_{i=3 k}^{L}\right\}_{k=0}^{(m-3) / 3}$ mapping $\psi_{i}^{L}: I_{i}=\left[t_{i}, t_{i+3}\right] \rightarrow\left[\hat{t}_{i}, \hat{t}_{i+3}\right]$ and fulfilling $t_{i+j}=\hat{\psi}_{i}^{L}\left(t_{i+j}\right)$, for $j=0,1,2,3$. Evidently if $\dot{\psi}_{i}^{L}>0\left(\right.$ as $\left.\hat{t}_{i}<\hat{t}_{i+1}\right)$ then $\psi_{i}^{L}: I_{i} \rightarrow \hat{I}_{i}=R g\left(\psi_{i}^{L}\right)$ (here $R g\left(\psi_{i}^{L}\right)$ denotes the range of $\left.\psi_{i}^{L}\right)$. On the other hand if $\psi_{i}^{L}$ is not injective we may also have $\psi_{i}^{L}: I_{i} \rightarrow \hat{I}_{i} \subset R g\left(\psi_{i}^{L}\right)$. In order to construct the composition $\hat{\gamma}_{i}^{L} \circ \psi_{i}^{L}$ as a well-defined function, each domain of $\hat{\gamma}_{i}^{L}$ is here understood as naturally extendable from $\hat{I}_{i}$ to $\mathbb{R}$. Such adjusted Lagrange piecewise-cubics denoted as $\check{\gamma}_{i}^{L}$ satisfy $\left.\check{\gamma}_{i}^{L}\right|_{\hat{I}_{i}}=\hat{\gamma}_{i}^{L}$. The following result holds (see e.g. [7,9] or [19]):

Theorem 1.4. Assume $\gamma \in C^{4}([0, T])$ be a regular curve in $\mathbb{E}^{n}$ sampled admissibly (see (1)). For $\hat{\gamma}^{L}$ and $\lambda=1$ in (3) each mapping $\psi_{i}^{L}$ is a $C^{\infty}$ reparameterization of $I_{i}$ into $\hat{I}_{i}$ and we have (uniformly over $[0, T]$ ):

$$
\gamma-\hat{\gamma}_{i}^{L} \circ \psi_{i}^{L}=O\left(\delta_{m}^{4}\right)
$$

In the remaining cases of $\lambda \in[0,1)$ from (3) let $\gamma$ be sampled more-or-less uniformly (see (2)). Then for each mapping $\psi_{i}^{L}$ combined with $\check{\gamma}_{i}^{L}$ the following holds (uniformly over $[0, T]$ ):

$$
\gamma-\check{\gamma}_{i}^{L} \circ \psi_{i}^{L}=O\left(\delta_{m}\right)
$$

Both (4) and (5) are sharp within the class of $\gamma \in C^{4}([0, T])$ and within a given family of admitted samplings, assumed here as either (1) or (2), respectively. By the latter we understand the existence of at least one $\gamma_{0} \in C^{4}([0, T])$ ) 
and some admissible (or more-or-less uniform) sampling $\mathcal{T}_{0}$ for which $\alpha(1)=4$ in (4) (or $\alpha(\lambda)=1$ for $\lambda \in[0,1)$ in (5)) are sharp according to Definition 1.3 - see also [9] or [12]. Note that $\psi^{L}$ as a track-sum of $\left\{\psi_{i=3 k}^{L}\right\}_{k=0}^{(m-3) / 3}$ defines a piecewise $C^{\infty}$ mapping of $I$ into $\mathbb{R}$ at least continuous at $\mathcal{T}$. If $\psi^{L}$ is a reparameterization (e.g. always holding asymptotically for $\lambda=1$ ) then $\psi^{L}: I \rightarrow \hat{I}$. In particular for $\lambda=1$ we also have $d(\gamma)-d\left(\hat{\gamma}^{L}\right)=O\left(\delta_{m}^{4}\right)$ - see [19]. In contrast, the injectivity of $\psi_{i}^{L}$ and length estimation for $\lambda \in[0,1)$ has not been so far examined.

In this paper we introduce two sufficient conditions enforcing each $\psi_{i}^{L}: I_{i} \rightarrow$ $\hat{I}_{i}$ to be injective, for $\lambda \in[0,1)$ governing the exponential parameterization $(3)$. These two conditions are represented by the inequalities (6) and (7). In the next step, Theorem 2.1 is established (the main result of this paper) to formulate several sufficient conditions enforcing (6) and (7) to hold asymptotically. Noticeably all derived conditions stipulating asymptotically the injectivity of $\psi^{L}$ are independent from $\gamma$ and apply to any fixed $\lambda \in[0,1)$ and to any preselected $\beta_{0}$-more-or-less-uniform samplings (i.e. to any $0<\beta_{0}<1$ fixed a priori). Additionally, all re-transformed algebraic constraints established here are visualized with the aid of $3 D$ plots in Mathematica (see [22]). The conditions can also be exploited once the incomplete information about samplings is available such as a priori knowledge of the respective upper and lower bounds for each triples $\left(M_{i m}, N_{i m}, P_{i m}\right)$ characterizing $\mathcal{T}$ as specified in (8) - see also Remark 3.1. The examples illustrate Theorem 2.1 and the relevance of this work (see Example 1). The conjecture concerning the sharp convergence rate $\alpha(\lambda)=2$ in length estimation $d(\gamma)-d\left(\hat{\gamma}^{L}\right)=O\left(\delta_{m}^{\alpha(\lambda)}\right)$ (combined with (3) for all $\lambda \in[0,1)$ yielding $\dot{\phi}>0$ ) is tested numerically (see Example 2 and Remark 3.2).

\section{Sufficient Conditions for Injectivity of $\psi_{i}^{L}$}

In this section we establish and discuss the asymptotic character (i.e. applicable for $m$ sufficiently large) of two sufficient conditions enforcing $\psi_{i}^{L}$ to be a genuine reparameterization of $I_{i}$ into $\hat{I}_{i}$ based on multidimensional reduced data $Q_{m}$.

Evidently the positivity of the quadratic $\dot{\psi}_{i}^{L}(t)=a_{i} t^{2}+b_{i} t+c_{i}$ over $I_{i}$ is e.g. guaranteed (for both sparse and dense data $Q_{m}$ ) provided if e.g. either (6) or (7) hold:

$$
\begin{aligned}
& a_{i}<0 \quad \text { and } \quad \dot{\psi}_{i}^{L}\left(t_{i}\right)>0 \quad \text { and } \quad \dot{\psi}_{i}^{L}\left(t_{i+3}\right)>0, \\
& a_{i}>0 \quad \text { and } \quad \dot{\psi}_{i}^{L}\left(-\frac{b_{i}}{2 a_{i}}\right)>0 \text {. }
\end{aligned}
$$

Noticeably, any admissible sampling (1) can be characterized as follows:

$$
t_{i+1}-t_{i}=M_{i m} \delta_{m}, \quad t_{i+2}-t_{i+1}=N_{i m} \delta_{m} \text { and } t_{i+3}-t_{i+2}=P_{i m} \delta_{m},
$$

where $0<M_{i m}, N_{i m}, P_{i m} \leq 1$. The main theoretical contribution of this paper reads as: 
Theorem 2.1. Let $\gamma \in C^{3}([0, T])$ be sampled $\beta_{0}$-more-or-less uniformly (see Definition (1.2)) with knots $\mathcal{T}$ represented by (8). For data $Q_{m}$ combined with exponential parameterization (3) (with any fixed $\lambda \in[0,1)$ ) the condition (6) yielding each $\psi_{i}^{L}: I \rightarrow \hat{I}_{i}$ as a reparameterization holds asymptotically, if the following three inequalities are satisfied for sufficiently large $m$ :

$$
\begin{gathered}
\frac{1}{P_{i m}+N_{i m}+M_{i m}}\left(\frac{P_{i m}^{\lambda-1}-N_{i m}^{\lambda-1}}{P_{i m}+N_{i m}}-\frac{N_{i m}^{\lambda-1}-M_{i m}^{\lambda-1}}{N_{i m}+M_{i m}}\right) \leq \rho<0, \\
M_{i m}^{\lambda-1}-\frac{\left(N_{i m}^{\lambda-1}-M_{i m}^{\lambda-1}\right) M_{i m}}{N_{i m}+M_{i m}}+\frac{\left(P_{i m}^{\lambda-1}-N_{i m}^{\lambda-1}\right) M_{i m}\left(N_{i m}+M_{i m}\right)}{\left(P_{i m}+N_{i m}\right)\left(P_{i m}+N_{i m}+M_{i m}\right)} \\
-\frac{\left(N_{i m}^{\lambda-1}-M_{i m}^{\lambda-1}\right) M_{i m}}{P_{i m}+N_{i m}+M_{i m}} \geq \rho_{1}>0, \\
P_{i m}^{\lambda-1}-\frac{\left(N_{i m}^{\lambda-1}-M_{i m}^{\lambda-1}\right) P_{i m}\left(P_{i m}+N_{i m}\right)}{\left(N_{i m}+M_{i m}\right)\left(P_{i m}+N_{i m}+M_{i m}\right)}+\frac{P_{i m}\left(P_{i m}^{\lambda-1}-N_{i m}^{\lambda-1}\right)}{P_{i m}+N_{i m}+M_{i m}} \\
+\frac{P_{i m}\left(P_{i m}^{\lambda-1}-N_{i m}^{\lambda-1}\right)}{P_{i m}+N_{i m}} \geq \rho_{2}>0,
\end{gathered}
$$

with fixed $\rho<0, \rho_{1}>0$ and $\rho_{2}>0$ but arbitrary small. Similarly, the condition (7) enforcing $\dot{\psi}_{i}^{L}>0$ holds asymptotically if the following two inequalities are met for sufficiently large $m$ :

$$
\begin{aligned}
& \frac{1}{P_{i m}+N_{i m}+M_{i m}}\left(\frac{P_{i m}^{\lambda-1}-N_{i m}^{\lambda-1}}{P_{i m}+N_{i m}}-\frac{N_{i m}^{\lambda-1}-M_{i m}^{\lambda-1}}{N_{i m}+M_{i m}}\right) \geq \rho_{3}>0, \\
& M_{i m}^{\lambda-1}+\frac{\left(N_{i m}^{\lambda-1}-M_{i m}^{\lambda-1}\right)\left(2 N_{i m}+M_{i m}\right)}{3\left(N_{i m}+M_{i m}\right)}-\frac{\left(N_{i m}^{\lambda-1}-M_{i m}^{\lambda-1}\right)^{2}}{3\left(N_{i m}+M_{i m}\right)} \\
& \cdot \frac{\left(P_{i m}+N_{i m}\right)\left(P_{i m}+N_{i m}+M_{i m}\right)}{\left(P_{i m}^{\lambda-1}-N_{i m}^{\lambda-1}\right)\left(N_{i m}+M_{i m}\right)-\left(N_{i m}^{\lambda-1}-M_{i m}^{\lambda-1}\right)\left(P_{i m}+N_{i m}\right)} \\
& -\left[\frac{\left(P_{i m}^{\lambda-1}-N_{i m}^{\lambda-1}\right)\left(N_{i m}+M_{i m}\right)-\left(N_{i m}^{\lambda-1}-M_{i m}^{\lambda-1}\right)\left(P_{i m}+N_{i m}\right)}{\left(N_{i m}+M_{i m}\right)\left(P_{i m}+N_{i m}\right)\left(P_{i m}+N_{i m}+M_{i m}\right)}\right] \\
& \cdot \frac{\left(N_{i m}^{2}+N_{i m} M_{i m}+M_{i m}^{2}\right)}{3} \geq \rho_{4}>0 \text {, }
\end{aligned}
$$

where constants $\rho_{3}>0$ and $\rho_{4}>0$ are fixed and small.

Proof. Newton interpolation formula (see [1]) based on divided differences of $\psi_{i}^{L}$ yields over $I_{i}$ :

$$
\begin{aligned}
\psi_{i}^{L}(t)= & \psi_{i}^{L}\left(t_{i}\right)+\psi_{i}^{L}\left[t_{i}, t_{i+1}\right]\left(t-t_{i}\right)+\psi_{i}^{L}\left[t_{i}, t_{i+1}, t_{i+2}\right]\left(t-t_{i}\right)\left(t-t_{i+1}\right) \\
& +\psi_{i}^{L}\left[t_{i}, t_{i+1}, t_{i+2}, t_{i+3}\right],
\end{aligned}
$$

which for each $t \in I_{i}$ renders $\dot{\psi}_{i}^{L}(t)=$

$$
\begin{gathered}
\psi_{i}^{L}\left[t_{i}, t_{i+1}\right]+\psi_{i}^{L}\left[t_{i}, t_{i+1}, t_{i+2}\right]\left(2 t-t_{i}-t_{i+1}\right)+\psi_{i}^{L}\left[t_{i}, t_{i+1}, t_{i+2}, t_{i+3}\right] \\
\cdot\left(\left(t-t_{i+1}\right)\left(t-t_{i+2}\right)+\left(t-t_{i}\right)\left(t-t_{i+2}\right)+\left(t-t_{i}\right)\left(t-t_{i+1}\right)\right) .
\end{gathered}
$$


We recall now the proof of (18) (see [9] or [12]) since it is vital for further arguments. As $\gamma$ is regular it can be assumed to be parameterized by arc-length rendering $\|\dot{\gamma}(t)\|=1$, for $t \in[0, T]$ (see [2]). The latter due to $1 \equiv\|\dot{\gamma}(t)\|^{2}=\langle\dot{\gamma}(t) \mid \dot{\gamma}(t)\rangle$ results in $0 \equiv\left(\|\dot{\gamma}(t)\|^{2}\right)^{\prime}=2\langle\dot{\gamma}(t) \mid \ddot{\gamma}(t)\rangle$ over $t \in[0, T]$. The orthogonality of $\dot{\gamma}$ and $\ddot{\gamma}$ nullifies certain terms in the expression (for $j=i+k$ with $k=0,1,2$ and any $\lambda \in[0,1])$ :

$\hat{t}_{j+1}-\hat{t}_{j}=\left\|q_{j+1}-q_{j}\right\|^{\lambda}=\left\|\gamma\left(t_{j+1}\right)-\gamma\left(t_{j}\right)\right\|^{\lambda}=\left\langle\gamma\left(t_{j+1}\right)-\gamma\left(t_{j}\right) \mid \gamma\left(t_{j+1}\right)-\gamma\left(t_{j}\right)\right\rangle^{\lambda}$

once Taylor expansion for $\gamma \in C^{3}$ is used:

$$
\gamma\left(t_{j+1}\right)-\gamma\left(t_{j}\right)=\left(t_{j+1}-t_{j}\right) \dot{\gamma}\left(t_{j}\right)+\frac{\left(t_{j+1}-t_{j}\right)^{2}}{2} \ddot{\gamma}\left(t_{j}\right)+O\left(\left(t_{j+1}-t_{j}\right)^{2}\right) .
$$

Indeed, upon substituting (16) into (15) and exploiting $\langle\dot{\gamma}(t) \mid \ddot{\gamma}(t)\rangle=0$ one obtains:

$$
\hat{t}_{j+1}-\hat{t}_{j}=\left(t_{j+1}-t_{j}\right)^{\lambda}\left(1+O\left(\left(t_{j+1}-t_{j}\right)^{2}\right)\right)^{\frac{\lambda}{2}} .
$$

For any admissible samplings the constants in the term $O\left(\left(t_{j+1}-t_{j}\right)^{2}\right)$ depend on the third derivative of $\gamma$ which is bounded over $[0, T]$ as $\gamma \in C^{3}$. Again Taylor Th. applied to the function $f(x)=(1+x)^{\frac{\lambda}{2}}$ at $x_{0}=0$ yields for all $x \in[-\varepsilon, \varepsilon]=I_{\varepsilon}$ (with some fixed $\varepsilon>0$ ) the existence of some $\xi_{x}$ satisfying $\left|\xi_{x}\right|<|x|$ such that $f(x)=1+\frac{\lambda}{2} x+\frac{\lambda}{4}\left(\frac{\lambda}{2}-1\right)\left(1+\xi_{x}\right)^{\frac{\lambda}{2}-2}$. For $0<\varepsilon<1$ we exclude the singularity of $\tau\left(\xi_{x}\right)=\left(1+\xi_{x}\right)^{\frac{\lambda}{2}-2}$ at $\xi_{x}=-1$ (with $\lambda \in[0,1]$ ) which forces $\tau$ to be bounded over $I_{\varepsilon}$. Thus for $\left|\xi_{x}\right|<|x| \leq \varepsilon<1$ we have $f_{1}(x)=1+\frac{\lambda}{2} x+O\left(x^{2}\right)$ - the constant standing along $x^{2}$ depends now on $\lambda$ (which is fixed). Take now $x=O\left(\left(t_{j+1}-t_{j}\right)^{2}\right)$ determined in (17) which is asymptotically small (for $m$ large) due to the admissibility condition (1) and thus separated from -1 . Hence the second-divided differences of $\psi_{i}^{L}$ satisfy (with $k=0,1,2$ ):

$\psi_{i}^{L}\left[t_{i+k}, t_{i+k+1}\right]=\frac{\hat{t}_{i+k+1}-\hat{t}_{i+k}}{t_{i+k+1}-t_{i+k}}=\left(t_{i+k+1}-t_{i+k}\right)^{\lambda-1}+O\left(\left(t_{i+k+1}-t_{i+k}\right)^{1+\lambda}\right)$.

Thus, by (8) and (18) one obtains for each $\lambda \in[0,1]$ and $k=0,1,2$ the following formula for the second divided differences of $\psi_{i}^{L}$ (needed also in (15)):

$$
\psi_{i}^{L}\left[t_{i+k}, t_{i+k+1}\right]=R_{i m k}^{\lambda-1} \delta_{m}^{\lambda-1}+O\left(\delta_{m}^{1+\lambda}\right),
$$

with $R_{i m 0}=M_{i m}, R_{i m 1}=N_{i m}$ and $R_{i m 2}=P_{i m}$. Furthermore still by (18) combined with $0<\left(t_{i+l+1}-t_{i+l}\right)\left(t_{i+2}-t_{i}\right)^{-1} \leq 1$ (for $l=0,1$ ) and telescoped $t_{i+2}-t_{i}=\left(t_{i+2}-t_{i+1}\right)+\left(t_{i+1}-t_{i}\right)$ the third-divided difference of $\psi_{i}^{L}$ is equal to $\psi_{i}^{L}\left[t_{i}, t_{i+1}, t_{i+2}\right]$

$$
\begin{aligned}
& =\frac{\left(t_{i+2}-t_{i+1}\right)^{\lambda-1}-\left(t_{i+1}-t_{i}\right)^{\lambda-1}}{t_{i+2}-t_{i}}+\frac{O\left(\left(t_{i+2}-t_{i+1}\right)^{1+\lambda}\right)+O\left(\left(t_{i+1}-t_{i}\right)^{1+\lambda}\right)}{t_{i+2}-t_{i}} \\
& =\frac{N_{i m}^{\lambda-1} \delta_{m}^{\lambda-1}-M_{i m}^{\lambda-1} \delta_{m}^{\lambda-1}}{\left(N_{i m}+M_{i m}\right) \delta_{m}}+O\left(\frac{\left(t_{i+2}-t_{i+1}\right)^{1+\lambda}}{t_{i+2}-t_{i}}\right)+O\left(\frac{\left(t_{i+1}-t_{i}\right)^{1+\lambda}}{t_{i+2}-t_{i}}\right) \\
& =\frac{N_{i m}^{\lambda-1}-M_{i m}^{\lambda-1}}{N_{i m}+M_{i m}} \delta_{m}^{\lambda-2}+O\left(\left(t_{i+2}-t_{i+1}\right)^{\lambda}\right)+O\left(\left(t_{i+1}-t_{i}\right)^{\lambda}\right)
\end{aligned}
$$


A similar argument leads to:

$\psi_{i}^{L}\left[t_{i+1}, t_{i+2}, t_{i+3}\right]=\frac{P_{i m}^{\lambda-1}-N_{i m}^{\lambda-1}}{P_{i m}+N_{i m}} \delta_{m}^{\lambda-2}+O\left(\left(t_{i+3}-t_{i+2}\right)^{\lambda}\right)+O\left(\left(t_{i+2}-t_{i+1}\right)^{\lambda}\right)$.

Hence by $(20)$ and $(21)$ (for $l=0,1)$ the third divided differences of $\psi_{i}^{L}$ (needed in (15)) read as:

$$
\psi_{i}^{L}\left[t_{i+l}, t_{i+l+1}, t_{i+l+2}\right]=\frac{R_{i m(l+1)}^{\lambda-1}-R_{i m l}^{\lambda-1}}{R_{i m(l+1)}+R_{i m l}} \delta_{m}^{\lambda-2}+O\left(\delta_{m}^{\lambda}\right) .
$$

Coupling again (20) and (21) with telescoped $t_{i+3}-t_{i}=\left(t_{i+3}-t_{i+2}\right)+\left(t_{i+2}-\right.$ $\left.t_{i+1}\right)+\left(t_{i+1}-t_{i}\right)$ and $0<\left(t_{i+l+1}-t_{i+l}\right)\left(t_{i+3}-t_{i}\right)^{-1}<1$ reduces the fourth divided difference of $\psi_{i}^{L}$ into:

$$
\begin{aligned}
\psi_{i}^{L}\left[t_{i}, t_{i+1}, t_{i+2}, t_{i+3}\right]= & \frac{\frac{P_{i m}^{\lambda-1}-N_{i m}^{\lambda-1}}{P_{i m}+N_{i m}}-\frac{N_{i m}^{\lambda-1}-M_{i m}^{\lambda-1}}{N_{i m}+M_{i m}}}{t_{i+3}-t_{i}} \delta_{m}^{\lambda-2} \\
& +\sum_{l=0}^{2} O\left(\frac{\left(t_{i+l+1}-t_{i+l}\right)^{\lambda}}{t_{i+3}-t_{i}}\right)
\end{aligned}
$$

which ultimately yields $\psi_{i}^{L}\left[t_{i}, t_{i+1}, t_{i+2}, t_{i+3}\right]$

$$
=\frac{1}{P_{i m}+N_{i m}+M_{i m}}\left(\frac{P_{i m}^{\lambda-1}-N_{i m}^{\lambda-1}}{P_{i m}+N_{i m}}-\frac{N_{i m}^{\lambda-1}-M_{i m}^{\lambda-1}}{N_{i m}+M_{i m}}\right) \delta_{m}^{\lambda-3}+O\left(\delta_{m}^{\lambda-1}\right) .
$$

The proof of $(23)$ relies on $O\left(\frac{\left(t_{i+l+1}-t_{i+l}\right)^{\lambda}}{t_{i+3}-t_{i}}\right)=O\left(\left(t_{i+l+1}-t_{i+l}\right)^{\lambda-1}\right)=O\left(\delta_{m}^{\lambda-1}\right)$. The second step resorts to more-or-less uniformity (3) of admitted samplings $\mathcal{T}$ for any $\lambda \in[0,1)($ as $\lambda-1<0)$. However, to keep all constants in $O\left(\delta_{m}^{\lambda-1}\right)$ from (23) as independent from each representative of (3) from now on we admit only $\beta_{0}$-more-or-less uniform samplings for some fixed $0<\beta_{0} \leq 1$ (see Definition 1.3). The latter permits to exploit the inequality $\left|\left(t_{i+l+1}-t_{i+l}\right)^{\lambda-1}\right| \leq \beta_{0}^{\lambda-1} \delta_{m}^{\lambda-1}$ to justify (23) with constants in $O\left(\delta_{m}^{\lambda-1}\right)$ depending on $\gamma$ and $\lambda$ (but not on samplings $\mathcal{T}$ ).

Recalling now that $\dot{\psi}_{i}^{L}(t)=a_{i} t^{2}+b_{i} t+c_{i}$ over $I_{i}$, by (15) we have:

$$
\begin{aligned}
a_{i}= & 3 \psi_{i}\left[t_{i}, t_{i+1}, t_{i+2}, t_{i+3}\right], \\
b_{i}= & 2 \psi_{i}\left[t_{i}, t_{i+1}, t_{i+2}\right]-2 \psi_{i}\left[t_{i}, t_{i+1}, t_{i+2}, t_{i+3}\right]\left(t_{i+2}+t_{i+1}+t_{i}\right), \\
c_{i}= & \psi_{i}\left[t_{i}, t_{i+1}\right]-\psi_{i}\left[t_{i}, t_{i+1} t_{i+2}\right]\left(t_{i}+t_{i+1}\right) \\
& +\psi_{i}\left[t_{i}, t_{i+1}, t_{i+2}, t_{i+3}\right]\left(t_{i} t_{i+1}+t_{i+1} t_{i+2}+t_{i} t_{i+2}\right) .
\end{aligned}
$$

In the next steps both conditions (6) and (7) enforcing $\dot{\psi}_{i}^{L}>0$ (for arbitrary $m$ ) are transformed into their asymptotic analogues applicable for sufficiently large $m$ (i.e. for $Q_{m}$ sufficiently dense). This will ultimately complete the proof of Theorem 2.1. 
In doing so, both conditions (6) and (7) are reformulated into asymptotic counterparts expressed in terms of $\left(M_{i m}, N_{i m}, P_{i m}\right)$ (see Theorem 2.1). To save space only the first inequality from (6) i.e. $a_{i}<0$ is fully addressed here (which automatically covers both (i) and (iv) - see (9) and (12)). The remaining more complicated cases (ii), (iii) and (v) (listed below) are supplemented with the final asymptotic formulas (10), (11) and (13). The proof of the latter shall be given in the full journal version of this paper.

(i) By (24) the first inequality $a_{i}<0$ from (6) amounts to $\psi_{i}^{L}\left[t_{i}, t_{i+1}, t_{i+2}\right.$, $\left.t_{i+3}\right]<0$ which in turn by $(23)$ holds subject to:

$$
\begin{aligned}
& \left(\frac{P_{i m}^{\lambda-1}-N_{i m}^{\lambda-1}}{\left(P_{i m}+N_{i m}\right)\left(P_{i m}+N_{i m}+M_{i m}\right)}-\frac{N_{i m}^{\lambda-1}-M_{i m}^{\lambda-1}}{\left(N_{i m}+M_{i m}\right)\left(P_{i m}+N_{i m}+M_{i m}\right)}\right) \delta_{m}^{\lambda-3} \\
& \quad+O\left(\delta_{m}^{\lambda-1}\right)<0
\end{aligned}
$$

for $\left(M_{i m}, N_{i m}, P_{i m}\right) \in\left[\beta_{0}, 1\right]^{3}$. Asymptotically, for fixed $\lambda \in[0,1)$ the slowest term determining the sign of (25) accompanies $\delta_{m}^{\lambda-3}$ and reads as (for all $\beta_{0^{-}}$ more-or-less uniform samplings):

$$
\theta_{1}\left(M_{i m}, N_{i m}, P_{i m}\right)=\frac{1}{P_{i m}+N_{i m}+M_{i m}}\left(\frac{P_{i m}^{\lambda-1}-N_{i m}^{\lambda-1}}{P_{i m}+N_{i m}}-\frac{N_{i m}^{\lambda-1}-M_{i m}^{\lambda-1}}{N_{i m}+M_{i m}}\right),
$$

provided $\theta_{1}$ is not of any order $\Theta\left(\delta_{m}^{2+\varepsilon}\right)$ with $\varepsilon \geq 0$. A possible sufficient condition guaranteeing the latter is to require:

$$
\theta_{1}\left(M_{i m}, N_{i m}, P_{i m}\right) \leq \rho<0,
$$

to hold for any fixed $\rho<0$. Evidently (26) amounts to the first inequality (9) assumed to hold in Theorem 2.1 in order to enforce in turn asymptotically the first inequality in (6) (for any fixed $\lambda \in[0,1)$ ).

(ii) A similar but longer argument shows that (upon combining (8), (15), (19), (22) and (23)) the asymptotic fulfillment of the second inequality from (6) i.e. $\dot{\psi}_{i}^{L}\left(t_{i}\right)>0$ is met subject to (10) satisfied for any fixed, but arbitrary small $\rho_{1}>0$ and sufficiently large $m$.

(iii) The third inequality $\dot{\psi}_{i}^{L}\left(t_{i+3}\right)>0$ determining (6) maps analogously into its asymptotic counterpart (11) assumed to be fulfilled for an arbitrary but fixed $\rho_{2}>0$ and $m$ sufficiently large.

(iv) Clearly the proof of (9) yields a symmetric sufficient condition for $\underline{a_{i}>0}$ (representing the first inequality in (7)) to hold asymptotically. The latter coincides with (12) stipulated to be satisfied by any fixed $\rho_{3}>0$, subject to $m$ getting large.

(v) The reformulation of $\kappa_{i m}=\dot{\psi}_{i}^{L}\left(\frac{-b_{i}}{2 a_{i}}\right)>0$ from (7) into (13) (assumed to hold for any fixed $\rho_{4}>0$ and sufficiently large $m$ ) involves a more intricate treatment (it is omitted here).

The asymptotic conditions established in Theorem 2.1 in the form of specific inequalities depend (for each $i$ ) exclusively on triples $\left(M_{i m}, N_{i m}, M_{i m}\right) \in\left[\beta_{0}, 1\right]^{3}$ 
and fixed $\lambda \in[0,1)$ (not on curve $\gamma$ ). Consequently, they can all be also visualized geometrically in $3 D$ for each $i=3 k$ and $\lambda \in[0,1)$ as well as for any regular curve $\gamma$. Several examples with $3 D$ plots are presented in Sect. 3 with the aid of Mathematica Package [22].

We note that all asymptotic conditions from Theorem 2.1 can be extended to their $2 D$ analogues (with extra argument used establishing in fact a new theorem) which in turn can be visualized in more appealing $2 D$ plots. Again it is omitted here as exceeding the scope of this paper.

Recall that uniform sampling, for which $M_{i m}=N_{i m}=P_{i m}=1$ (i.e. where $\left.\beta_{0}=1\right)$ combined with $\lambda \in[0,1)$ or $\lambda=1$ with (1) both yield $\dot{\psi}_{i}^{L}=1+$ $O\left(\delta_{m}^{2}\right)>0($ see [9] and [19])). Noticeably, conditions (10), (11) and (13) are met for either $\lambda=1$ or $\mathcal{T}$ uniform and $\lambda \in[0,1)$. In contrast none of (9) or (12) (participating in either (6) or (7)) holds for the above two eventualities. A possible remedy to incorporate these two special cases in adjusted asymptotic representations of either $a_{i}>0$ or $a_{i}<0$ is to apply the fourth-order Taylor expansion for $\gamma \in C^{4}$ - see (16). The analysis (left out here) yields a modified condition for $a_{i}>0$ (and thus for $a_{i}<0$ ), this time hinging not only on triples $\left(M_{i m}, N_{i m}, P_{i m}\right) \in\left[\beta_{0}, 1\right]^{3}, \lambda \in[0,1)$ but also on $\gamma$ curvature $\left\|\ddot{\gamma}\left(t_{i}\right)\right\|^{2}$ along $\mathcal{T}$ (see [9] and [19]) - here $\|\dot{\gamma}(t)\|=1$ as $\gamma$ is a regular curve and as such can be assumed to be parameterized by arc-length (see [2]). The latter may not always be given in advance. Alternatively, one could rely on a priori imposed restrictions on curvatures of $\gamma$ belonging to the prescribed family of admissible curves.

\section{Experimentation and Testing}

In this section first Theorem 2.1 is illustrated with some examples based on algebraic tests supported by $3 D$ plots generated in Mathematica (see Subsect. 3.1). Next the convergence rate $\alpha(\lambda)$ for $d(\gamma)-d\left(\hat{\gamma}^{L}\right)=O\left(\delta_{m}^{\alpha(\lambda)}\right)$ is numerically investigated. A special attention is given to $\lambda \in[0,1)$ yielding $\psi^{L}$ as a piecewise $C^{\infty}$ reparameterization of $[0, T]$ into $[0, \hat{T}]$ (see Subsect. 3.2 ).

In doing so, in a preliminary step, for a given fixed $\beta_{0}$ two families of $\beta_{0^{-}}$ more-or-less uniform samplings (27) and (29) are introduced. Next the fulfillment of the asymptotic sufficient conditions enforcing the injectivity of $\dot{\psi}^{L}>0$ (see Theorem 2.1) is examined for various $\lambda \in[0,1)$ and both samplings (27) and (29). In particular, the inequalities (9), (10), (11), (denoted in this section by $\left.(6)^{*}\right)$ and $(12),(13)$ (marked here with $\left.(7)^{*}\right)$ representing asymptotically in $3 D$ both (6) and (7) are tested for different sets of triples $\left(M_{i m}, N_{i m}, P_{i m}\right) \in\left[\beta_{0}, 1\right]^{3}$ characterizing either (27) or (29). The algebraic calculations performed herein (assuming $m$ is sufficiently large) are supplemented by geometrical visualizations with $3 D$ plots in Mathematica. At this point, we re-emphasize that the asymptotic conditions from Theorem 2.1 can be extended further into respective $2 D$ counterparts upon some laborious calculations. In return, the latter gives some advantage in visualizing more appealing $2 D$ (versus $3 D$ ) plots. To save the space the relevant theory and testing concerning this extra $2 D$ case are left out here. 
The second example reports on tests designed to numerically evaluate $\alpha(\lambda)$ in length estimation $d(\gamma)-d\left(\hat{\gamma}^{L}\right)=O\left(\delta^{\alpha(\lambda)}\right)$, for any $\lambda \in[0,1]$ yielding each $\psi_{i}^{L}$ as an injective function. The conjecture concerning $\alpha(\lambda)$ is proposed in Remark 3.2 based on our numerical results.

The tests reported here are performed for $2 D$ and $3 D$ curves $\gamma_{s p}, \gamma_{S}$ introduced in Example 2 (i.e. for $n=2,3$ ). However all established results with the accompanied experimentation are equally applicable to arbitrary multidimensional reduced data $Q_{m}=\left\{q_{i}\right\}_{i=0}^{m}$ with $q_{i}=\gamma\left(t_{i}\right) \in \mathbb{E}^{n}$.

\subsection{Testing Injectivity of $\psi^{L}$}

Example 1. Consider first the following family $\mathcal{T}_{1}$ of more-or-less uniform sampling (for geometrical distribution of $\left\{\gamma\left(t_{i}\right)\right\}_{i=0}^{15}$ with sampling (27) see also Fig. 3(a) and Fig. 4(a)):

$$
t_{i}=\left\{\begin{array}{lc}
\frac{i}{m}+\frac{1}{2 m}, & \text { for } \quad i=4 k+1, \\
\frac{i}{m}-\frac{1}{2 m}, & \text { for } i=4 k+3, \\
\frac{i}{m}, & \text { for } \quad i=2 k,
\end{array}\right.
$$

for which $K_{l}=\frac{1}{2}, K_{u}=\frac{3}{2}$ and $\beta_{1}=\frac{1}{3}$ (see Definition 1.2). Here $0 \leq i \leq m=3 k$, where $k \in\{1,2, \ldots\}$, so that $t_{0}=0$ and $t_{m}=T=1$. Upon resorting to (8) the following $3 D$ compact asymptotic representation $\mathcal{T}_{1}^{3 D}$ of $\mathcal{T}_{1}$ reads as (for $m=3 k)$ :

$$
\mathcal{T}_{1}^{3 D}=\left\{\left(1, \frac{1}{3}, \frac{1}{3}\right),\left(1,1, \frac{1}{3}\right),\left(\frac{1}{3}, 1,1\right),\left(\frac{1}{3}, \frac{1}{3}, 1\right),\left(1, \frac{1}{3}, \frac{2}{3}\right),\left(\frac{1}{3}, 1, \frac{2}{3}\right)\right\} .
$$

The last two points in (28) are generated for $m=3 k$ as $t_{m}=1$. We set $\beta_{0}=0.16$ and hence as $\beta_{0} \leq \beta_{1}$ the sampling (27) is also $\beta_{0}$-more-or-less uniform.

We also admit another $\beta_{0}$-more-or-less uniform sampling $\mathcal{T}_{2}$ defined according to (for geometrical spread of $\left\{\gamma\left(t_{i}\right)\right\}_{i=0}^{15}$ with sampling (29) see also Fig. 3(b) and Fig. 4(b)):

$$
t_{i}=\frac{i}{m}+\frac{(-1)^{i+1}}{3 m},
$$

with $K_{l}=\frac{1}{3}, K_{u}=\frac{5}{3}$ and $\beta_{2}=\frac{1}{5} \geq \beta_{0}$ (see Definition 1.2). Again we set $t_{0}=0$ and $t_{m}=T=1$ with $0 \leq i \leq m=3 k$, for $k \in\{1,2, \ldots\}$. By (8) the $3 D$ asymptotic form $\mathcal{T}_{2}^{3 D}$ of $(29)$ reads as:

$$
\mathcal{T}_{2}^{3 D}=\left\{\left(\frac{4}{5}, \frac{1}{5}, 1\right),\left(\frac{1}{5}, 1, \frac{1}{5}\right),\left(1, \frac{1}{5}, 1\right),\left(1, \frac{1}{5}, \frac{4}{5}\right),\left(\frac{1}{5}, 1, \frac{2}{5}\right)\right\} .
$$

The last two points in (30) come for $m=3 k$ as $t_{m}=1$ and the first point is due to $t_{0}=0$.

The inequalities (9), (10), (11) marked as (6)* (or (12) and (13) denoted by $(7)^{*}$ ) enforcing asymptotically (6) (or (7)) to hold are tested over $\left[\beta_{0}, 1\right]^{3}$ for both samplings (27) and (29). The fixed parameter $\lambda$ is set either to $\lambda=0.3$ or to $\lambda=0.9$ with $\rho=-0.001, \rho_{1}=\rho_{2}=0.05, \rho_{3}=0.001$ and $\rho_{4}=0.005$ - see Table 1 and Table 2 . The corresponding sets of triples $\left(M_{i m}, N_{i m}, P_{i m}\right) \in\left[\beta_{0}, 1\right]^{3}$ 
Table 1. Testing conditions (6) and (7) (implied asymptotically by $(6)^{*}$ and $\left.(7)^{*}\right)$ for sampling (27) (represented by (28)) and for $\lambda=0.3$ and $\lambda=0.9$ with $\rho=-0.001$, $\rho_{1}=0.05, \rho_{2}=0.05, \rho_{3}=0.001$ and $\rho_{4}=0.005$. Here $\mathbf{T}$ stands for true and $\mathbf{F}$ for false, respectively.

\begin{tabular}{c|c|c|c|c}
\hline$\lambda$ & \multicolumn{3}{|c|}{$\lambda=0.3$} & \multicolumn{2}{|c}{$\lambda=0.9$} \\
\hline \multirow{2}{*}{ Sampling $\mathcal{T}_{1}^{3 D}$} & \multicolumn{3}{|c}{ Conditions } \\
\cline { 2 - 5 } & $(6)^{*}$ & $(7)^{*}$ & $(6)^{*}$ & $(7)^{*}$ \\
\hline$\left(1, \frac{1}{3}, \frac{1}{3}\right)$ & $\mathbf{F}$ & $\mathbf{F}$ & $\mathbf{T}$ & $\mathbf{F}$ \\
\hline$\left(1,1, \frac{1}{3}\right)$ & $\mathbf{F}$ & $\mathbf{T}$ & $\mathbf{F}$ & $\mathbf{T}$ \\
\hline$\left(\frac{1}{3}, 1,1\right)$ & $\mathbf{F}$ & $\mathbf{T}$ & $\mathbf{F}$ & $\mathbf{T}$ \\
\hline$\left(\frac{1}{3}, \frac{1}{3}, 1\right)$ & $\mathbf{F}$ & $\mathbf{F}$ & $\mathbf{T}$ & $\mathbf{F}$ \\
\hline$\left(1, \frac{1}{3}, \frac{2}{3}\right)$ & $\mathbf{F}$ & $\mathbf{F}$ & $\mathbf{T}$ & $\mathbf{F}$ \\
\hline$\left(\frac{1}{3}, 1, \frac{2}{3}\right)$ & $\mathbf{F}$ & $\mathbf{T}$ & $\mathbf{F}$ & $\mathbf{T}$ \\
\hline
\end{tabular}

Table 2. Testing conditions (6) and (7) (implied asymptotically by $(6)^{*}$ and $\left.(7)^{*}\right)$ for sampling (29) (represented by (30)) and for $\lambda=0.3$ and $\lambda=0.9$ with $\rho=-0.001$, $\rho_{1}=0.05, \rho_{2}=0.05, \rho_{3}=0.001$ and $\rho_{4}=0.005$. Here $\mathbf{T}$ stands for true and $\mathbf{F}$ for false, respectively.

\begin{tabular}{c|c|c|c|c}
\hline$\lambda$ & \multicolumn{3}{|c|}{$\lambda=0.3$} & \multicolumn{2}{c}{$\lambda=0.9$} \\
\hline \multirow{2}{*}{ Sampling $\mathcal{T}_{2}^{3 D}$} & \multicolumn{4}{|c}{ Conditions } \\
\cline { 2 - 5 } & $(6)^{*}$ & $(7)^{*}$ & $(6)^{*}$ & $(7)^{*}$ \\
\hline$\left(\frac{4}{5}, \frac{1}{5}, 1\right)$ & $\mathbf{F}$ & $\mathbf{F}$ & $\mathbf{T}$ & $\mathbf{F}$ \\
\hline$\left(\frac{1}{5}, 1, \frac{1}{5}\right)$ & $\mathbf{F}$ & $\mathbf{T}$ & $\mathbf{F}$ & $\mathbf{T}$ \\
\hline$\left(1, \frac{1}{5}, 1\right)$ & $\mathbf{F}$ & $\mathbf{F}$ & $\mathbf{T}$ & $\mathbf{F}$ \\
\hline$\left(1, \frac{1}{5}, \frac{4}{5}\right)$ & $\mathbf{F}$ & $\mathbf{F}$ & $\mathbf{T}$ & $\mathbf{F}$ \\
\hline$\left(\frac{1}{5}, 1, \frac{2}{5}\right)$ & $\mathbf{F}$ & $\mathbf{T}$ & $\mathbf{F}$ & $\mathbf{T}$ \\
\hline
\end{tabular}

satisfying either $(6)^{*}$ or $(7)^{*}$ represent the respective solids $D_{\beta_{0}}^{\lambda} \subset\left[\beta_{0}, 1\right]^{3}$ plotted in $3 D$ by Mathematica as shown in Fig. 1 and Fig. 2.

Noticeably different points from $\mathcal{T}_{k}^{3 D}$, for $k=1,2$ may interchangeably satisfy one of the sufficient conditions enforcing either (6) or (7) to hold asymptotically. The latter is demonstrated in Table 1 and Table 2. Indeed for $\lambda=0.3$ all conditions from $(6)^{*}$ are not satisfied by both $\mathcal{T}_{k}^{3 D}$ (for $k=1,2$ ) as we have $\mathbf{F}$ in the respective columns of both Table 1 and Table 2. Moreover, the conditions from $(7)^{*}$ are only fulfilled by some points (not all) from $\mathcal{T}_{k}^{3 D}$. Consequently the injectivity of $\psi_{i}^{L}$ for either $\mathcal{T}_{1}^{3 D}$ or $\mathcal{T}_{2}^{3 D}$ is not guaranteed. Geometrically both $\mathcal{T}_{k}^{3 D}$ (for $k=1,2$ ) are not contained in the respective injectivity zones $D_{\beta_{0}}^{\lambda=0.3}$ (for either $(6)^{*}$ or $\left.(7)^{*}\right)$. In contrast for $\lambda=0.9$, a simple inspection of Table 1 and Table 2 reveals that all points from $\mathcal{T}_{k}^{3 D}$ (for $k=1,2$ ) can be split into two subsets each contained in the injectivity zones $D_{\beta_{0}}^{\lambda=0.9}$ determined by either $(6)^{*}$ 


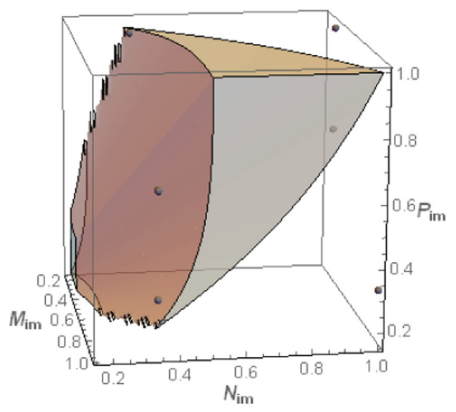

a)

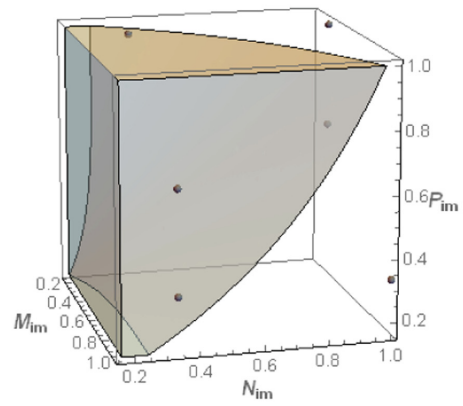

c)

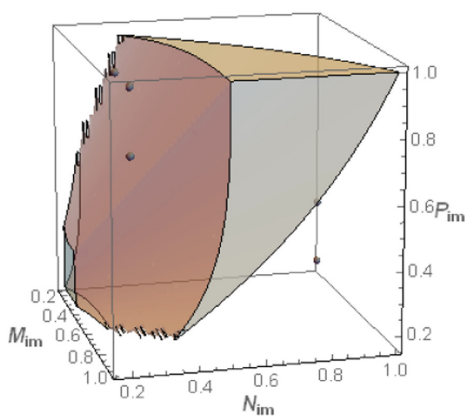

b)

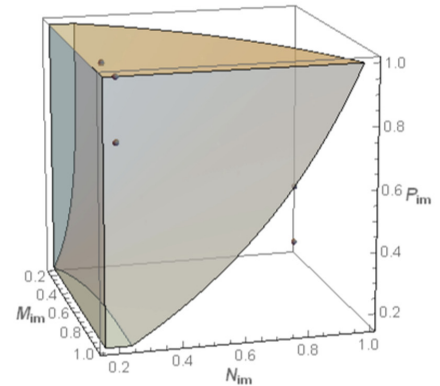

d)

Fig. 1. Condition (6) enforced asymptotically by $(6)^{*}$ visualized in $3 D$ plots as two solids $D_{\beta_{0}}^{\lambda} \subset\left[\beta_{0}, 1\right]^{3}$, for $\lambda=0.3$ or $\lambda=0.9$, respectively. Here $\beta_{0}=0.16$ with dotted points representing samplings: a) (27) mapped into (28) or b) (29) mapped into (30) both for $\lambda=0.3$ and samplings: c) (27) mapped into (28) or d) (29)mapped into (30) both for $\lambda=0.9$.

or by $(7)^{*}$, respectively. Algebraically the latter yields at least one $\mathbf{T}$ in the last two columns of all rows for both Table 1 and Table 2.

Remark 3.1. Note that if for a given family of $\beta_{0}$-more-or-less uniform samplings $\mathcal{T}_{\beta_{0}}$ the subfamily $\mathcal{T}_{\beta_{0}}^{\nu} \subset \mathcal{T}_{\beta_{0}}$ with extra constraints $\nu_{1} \leq M_{i m} \leq \nu_{2}, \nu_{3} \leq N_{i m} \leq$ $\nu_{4}$ and $\nu_{5} \leq P_{i m} \leq \nu_{6}$ (here $\nu=\left(\nu_{1}, \nu_{2}, \nu_{3}, \nu_{4}, \nu_{5}, \nu_{6}\right)$ ) is chosen one can also examine (for a fixed $\lambda \in[0,1)$ ) whether $I_{\nu}^{3 D} \subset D_{\beta_{0}}^{\lambda}$, where $I_{\nu}^{3 D}=\left(\nu_{1}, \nu_{2}\right) \times$ $\left(\nu_{3}, \nu_{4}\right) \times\left(\nu_{5}, \nu_{6}\right)$. By Theorem 2.1, should the latter holds the entire subfamily of $\mathcal{T}_{\beta_{0}}^{\nu}$ yields asymptotically $\psi_{i}^{L}$ as injective functions. The incomplete information on input samplings $\mathcal{T}$ carried by $\mathcal{T}_{\beta_{0}}^{\nu}$ can in certain situations accompany $Q_{m}$.

\subsection{Numerical Testing for Length Estimation}

We pass now to the experiments designed to investigate convergence rate $\alpha(\lambda)$ in length approximation by examining $d(\gamma)-d(\hat{\gamma})=O\left(\delta^{\alpha(\lambda)}\right)$ - see Definition 1.3. 
The coefficient $\alpha(\lambda)$ is estimated numerically by $\tilde{\alpha}(\lambda)$ which in turn is computed using a linear regression on the pairs $\left\{\left(\log (m),-\log \left(E_{m}\right)\right\}_{m=m_{\text {min }}}^{m=m_{\max }}\right.$, where $E_{m}=$ $\left|d(\gamma)-d\left(\hat{\gamma}^{L}\right)\right|$, for a given $m$. The slope $a$ of the regression line $y(x)=a x+b$ found in Mathematica with the aid of Normal[LinearModelFit[data]] yields $a=$ $\tilde{\alpha}(\lambda)$ forming a numerical estimate of $\alpha(\lambda)$.

Example 2. Consider a $2 D$ spiral $\gamma_{s p}:[0,1] \rightarrow \mathbb{E}^{2}$ (a regular curve with $\gamma_{s p}(0)=$ $(-0.2,0)$ and $\left.\gamma_{s p}(1)=(1.2,0)\right)$ :

$$
\gamma_{s p}(t)=((t+0.2) \cos (\pi(1-t)),(t+0.2) \sin (\pi(1-t))),
$$

and the so-called $3 D$ Steinmetz curve $\gamma_{S}:[0,1] \rightarrow \mathbb{E}^{3}$ (a regular closed curve with $\gamma_{S}(0)=\gamma_{S}(1)=(1,0,1.2)$ - see a dotted gray point in Fig. 4$)$ :

$$
\gamma_{S}(t)=\left(\cos (2 \pi t), \sin (2 \pi t), \sqrt{1.2^{2}-1.0^{2} \sin ^{2}(2 \pi t)}\right) .
$$

Both curves $\gamma_{s p}, \gamma_{S}$ (from (31) and (32)) sampled according to either (27) or (29) are plotted in Fig. 3 and Fig. 4, respectively. The numerical results assessing the estimate $\tilde{\alpha}(\lambda)$ of $\alpha(\lambda)$ (for $d(\gamma)-d\left(\hat{\gamma}^{L}\right)=O\left(\delta_{m}^{\alpha(\lambda)}\right)$ ) are presented in Table 3. Recall that here, a linear regression to compute $\tilde{\alpha}(\lambda)$ is applied to the collections of points $\left\{\left(\log (m),-\log \left(E_{m}\right)\right\}_{m_{\min }=120}^{m_{\max }=201}\right.$, with $E_{m}=\left|d(\gamma)-d\left(\hat{\gamma}^{L}\right)\right|$ and for various $\lambda \in\{0.3,0.7,0.9\}$. The results from Table 3 suggest that for all $\lambda \in\{0.3,0.7,0.9\}$ rendering $\dot{\psi}^{L}>0$ (e.g. the latter is guaranteed if Theorem 2.1 holds) one may expect $\lim _{m \rightarrow \infty} E_{m}=0$ with the quadratic convergence rate $\alpha(\lambda)=2 \approx \tilde{\alpha}(\lambda)$.

In fact the numerical results from Example 2 combined with (5) in conjunction with the argument used to prove $d(\gamma)-d\left(\hat{\gamma}^{L}\right)=O\left(\delta_{m}^{4}\right)$ for $\lambda=1$ (see [7]) or [19]) lead to expect $\alpha(\lambda)=2$ in $d(\gamma)-d\left(\hat{\gamma}^{L}\right)=O\left(\delta_{m}^{\alpha(\lambda)}\right)$, for all $\lambda \in[0,1)$ yielding $\psi^{L}$ as a piecewise $C^{\infty}$ reparametrization. The latter forms an open problem which can be stated as:

Remark 3.2. Assume $\gamma \in C^{4}([0, T])$ be a regular curve in $\mathbb{E}^{n}$ sampled more-orless uniformly (see Definition 1.2). For the interpolant $\hat{\gamma}^{L}$ and any $\lambda \in[0,1$ ) in (3) yielding each $\psi_{i}^{L}: I \rightarrow \hat{I}$ as a $C^{\infty}$ genuine reparameterization Example 2 suggests a sharp quadratic convergence rate in:

$$
d(\gamma)-d\left(\hat{\gamma}_{i}^{L} \circ \psi_{i}^{L}\right)=O\left(\delta_{m}^{2}\right)
$$

In particular if Theorem 2.1 holds (and $\beta_{0}$-more-or-less uniform samplings are used) the mapping $\psi^{L}$ is asymptotically a reparameterization which in turn hints to expect (33). Recall that by sharpness of (33) we understand the existence of at least one regular curve of class $C^{4}$ and of at least one samplings from $\mathcal{T}_{\beta_{0}}$ such that in (33) the convergence rate $\alpha(\lambda)$ has exactly order 2 (i.e. is not faster than quadratic). 


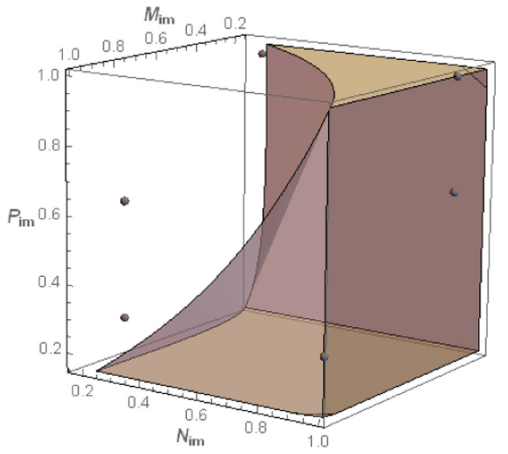

a)

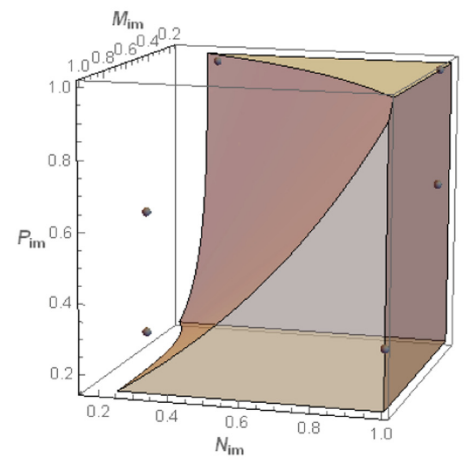

c)

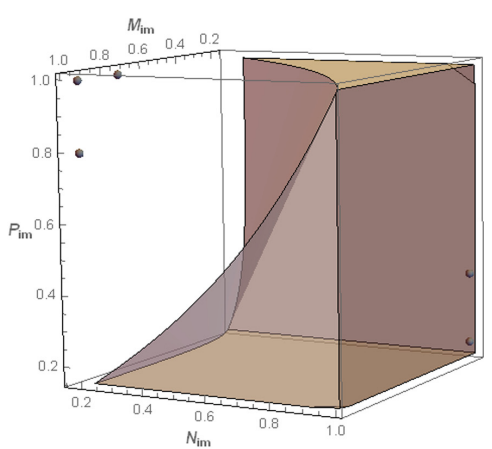

b)

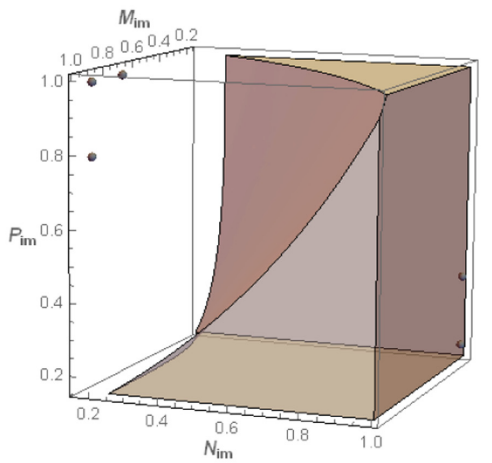

d)

Fig. 2. Condition (7) enforced asymptotically by $(7)^{*}$ visualized in $3 D$ plots as two solids $D_{\beta_{0}}^{\lambda} \subset\left[\beta_{0}, 1\right]^{3}$, for $\lambda=0.3$ or $\lambda=0.9$, respectively. Here $\beta_{0}=0.16$ with dotted points representing samplings: a) (27) mapped into (28) or b) (29) mapped into (30) both for $\lambda=0.3$ and samplings: c) (27) mapped into (28) or $d$ ) (29) mapped into (30) both for $\lambda=0.9$.

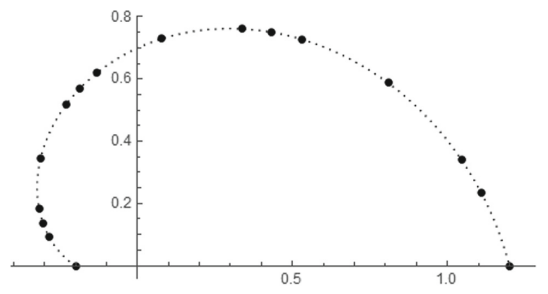

a)

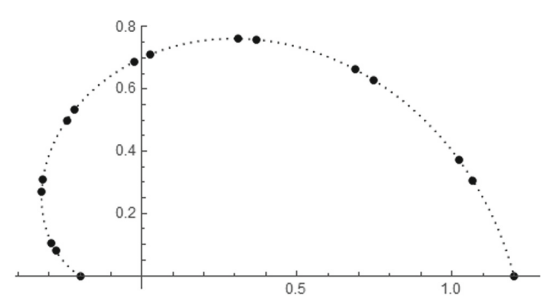

b)

Fig. 3. A spiral curve $\gamma_{s p}$ from (31) sampled according to: a) (27) or b) (29), for $m=15$. 


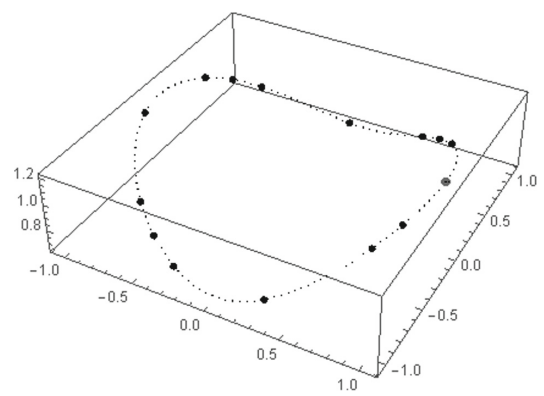

a)

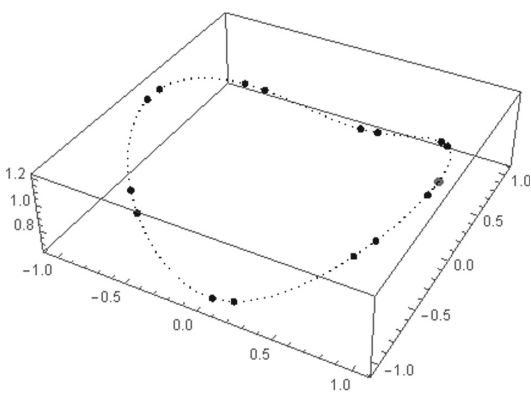

b)

Fig. 4. A Steinmetz curve $\gamma_{S}$ from (32) sampled according to: a) (27) or b) (29), for $m=15$ (with dotted gray point $\gamma_{S}(0)=\gamma_{S}(1)=(1,0,1.2)$ ).

Table 3. The numerical estimates of $\alpha(\lambda) \approx \tilde{\alpha}(\lambda)$ for a spiral $\gamma_{s p}$ from (31) and $a$ Steinmetz curve $\gamma_{S}$ from (32) computed for $m_{\min }=120 \leq m \leq m_{\max }=201$ and $\lambda \in\{0.3,0.7,0.9\}$. Here $\mathbf{T}$ stands for true and $\mathbf{F}$ for false, respectively.

\begin{tabular}{|c|c|c|c|c|c|}
\hline Curve & Sampling & $\lambda$ & $\mathrm{E}_{m=201}$ & $\alpha(\lambda) \approx \tilde{\alpha}(\lambda)$ & $(6)^{*}$ or $(7)^{*}$ \\
\hline \multirow{6}{*}{ (31) } & \multirow{3}{*}{$(27)$} & 0.3 & 0.0735200 & 0.044 & $\mathbf{F}$ \\
\hline & & 0.7 & 0.0000083 & 1.945 & $\mathbf{T}$ \\
\hline & & 0.9 & 0.0000016 & 1.885 & $\mathbf{T}$ \\
\hline & \multirow{3}{*}{ (29) } & 0.3 & 2.4619100 & -0.012 & $\mathbf{F}$ \\
\hline & & 0.7 & 0.0050445 & 0.007 & $\mathbf{F}$ \\
\hline & & 0.9 & 0.0000319 & 1.989 & $\mathbf{T}$ \\
\hline \multirow{6}{*}{$(32)$} & \multirow{3}{*}{ (27) } & 0.3 & 0.2036000 & 0.033 & $\mathbf{F}$ \\
\hline & & 0.7 & 0.0000897 & 2.015 & $\mathbf{T}$ \\
\hline & & 0.9 & 0.0000181 & 2.092 & $\mathbf{T}$ \\
\hline & \multirow{3}{*}{ (29) } & 0.3 & 6.7392400 & -0.009 & $\mathbf{F}$ \\
\hline & & 0.7 & 0.0132964 & -0.080 & $\mathbf{F}$ \\
\hline & & 0.9 & 0.0003419 & 1.985 & $\mathbf{T}$ \\
\hline
\end{tabular}

\section{Conclusions}

Fitting reduced data (see e.g. [3] or [16]) constitutes an important task in computer vision and graphics, engineering, microbiology, physics and other applications like medical image processing (e.g. for area, length and boundary estimation or trajectory planning) - see e.g. $[4,6,8,11,14,15,17,20]$ or [21].

Two sufficient conditions (6) and (7) are first formulated to ensure that the Lagrange piecewise-cubic $\psi^{L}:[0, T] \rightarrow[0, \hat{T}]$ (introduced in Sect. 1 ) is a genuine reparameterization. The latter applies to both sparse and dense reduced data $Q_{m}$. Here the unknown interpolation knots $\mathcal{T}$ are replaced by $\hat{\mathcal{T}}$ which in turn 
is determined by exponential parameterization (3) controlled by a single parameter $\lambda \in[0,1]$ and $Q_{m}$. The main contribution established in Theorem 2.1 (see Sect. 2) reformulates (6) and (7) into respective asymptotic representatives valid for sufficiently large $m$ (i.e. for $Q_{m}$ getting denser). These new transformed conditions (specified in Theorem 2.1) depend exclusively on $\lambda \in[0,1)$ and $\mathcal{T}$ characterized by (8) within the admitted class of $\beta_{0}$-more-or-less uniform samplings (see Definition 1.2) and apply to any regular curve $\gamma \in C^{3}([0, T])$ (with $0<T<\infty)$. Lastly, in Sect. 3 two illustrative examples are presented. The attached $3 D$ plots generated in Mathematica [22] illustrate the algebraic character of the asymptotic conditions justified in Theorem 2.1 (see Example 1). In addition, the numerical examination of the convergence rate in length estimation of interpolated $\gamma$ for $\lambda \in\{0.3,0.7,0.9\}$ are performed. Consequently, based on the latter the conjecture suggesting the quadratic convergence rate for $d(\gamma)-d\left(\hat{\gamma}^{L}\right)=O\left(\delta_{m}^{2}\right)$ is posed (see Example 2 and Remark 3.2), subject to the injectivity of $\psi^{L}$. At this point we remark that all asymptotic formulas from Theorem 2.1 are extendable to the corresponding inequalities expressed in $(x, y)$-variables. This can be achieved by converting first (with the aid of special homogeneous mapping) each triple $\left(M_{i m}, N_{i m}, P_{i m}\right)$ from (8) into a pair $\left(x\left(M_{i m}, N_{i m}, P_{i m}\right), y\left(M_{i m}, N_{i m}, P_{i m}\right)\right)$ and then by reformulating all conditions from Theorem 2.1, accordingly in terms of $(x, y)$. The satisfaction of such new conditions enforces (9), (10) and (11) or (12) and (13) asymptotically (and thus of (6) or (7)). It is a big advantage to reduce the illustrations from $3 D$ to more appealing $2 D$ analogues. We omit here the theoretical discussion and the geometrical insight of this $2 D$ extension of Theorem 2.1. Similarly, recall that only items (i) and (iv) (see Sect.2) are given here a full proof. In contrast, the final steps of proving (ii), (iii) and (v) are left out as treated later exhaustively in a journal version of this work (together with the mentioned above $2 D$ extension of Theorem 2.1).

Future work may include various interpolation schemes $\hat{\gamma}$ or $\phi$ based on $Q_{m}$ combined with either (3) or with other $\hat{\mathcal{T}}$ compensating the unknown knots $\mathcal{T}$ (see e.g. [3,10,13] or [16]). Searching for alternative sufficient conditions enforcing $\psi_{i}^{L}$ to be injective forms an interesting topic. Lastly the theoretical justification of (33) poses another open problem.

\section{References}

1. de Boor, C.: A Practical Guide to Spline. Springer, New York, 1985. https://www. researchgate.net/publication/200744645_A_Practical_Guide_to_Spline

2. do Carmo, M.P.: Differential Geometry of Curves and Surfaces. Prentice-Hall, Englewood Cliffs (1976). http://www2.ing.unipi.it/griff/files/dC.pdf

3. Epstein, M.P.: On the influence of parameterization in parametric interpolation. SIAM J. Numer. Anal. 13(2), 261-268 (1976). https://doi.org/10.1137/0713025

4. Farin, G.: Curves and Surfaces for Computer Aided Geometric Design. Academic Press, Cambridge (1993). https://www.sciencedirect.com/book/9780122490521/ curves-and-surfaces-for-computer-aided-geometric-design

5. Floater, M.S.: Chordal cubic spline interpolation is fourth-order accurate. IMA J. Numer. Anal. 25(1), 25-33 (2005). https://doi.org/10.1093/imanum/dri022 
6. Janik, M., Kozera, R., Kozioł, P.: Reduced data for curve modeling - applications in graphics, computer vision and physics. Adv. Sci. Technol. Res. J. 7(18), 28-35 (2013). https://doi.org/10.5604/20804075.1049599

7. Kozera, R.: Curve modeling via interpolation based on multidimensional reduced data. Stud. Inform. 25(4B(61)), 1-140 (2004). https://doi.org/10.21936/ si2004_v25.n4B

8. Kozera, R., Wilkołazka, M.: A natural spline interpolation and exponential parameterization for length estimation of curves. Proc. Amer. Inst. Phys. 1863(1), 4000101-400010-4 (2017). https://doi.org/10.1063/1.4992579

9. Kozera, R., Wilkołazka, M.: Convergence order in trajectory estimation by piecewise cubics and exponential parameterization. Math. Model. Anal. 24(1), 72-94 (2019). https://doi.org/10.3846/mma.2019.006

10. Kozera, R., Wilkołazka, M.: A note on modified Hermite interpolation. Math. Comput. Sci. 14, 223-239 (2020). https://doi.org/10.1007/s11786-019-00434-3

11. Kozera, R., Noakes, L.: $C^{1}$ interpolation with cumulative chord cubics. Fundam. Inform. 61(3), 285-301 (2004). https://dl.acm.org/doi/abs/10.5555/1031956. 1031962

12. Kozera, R., Noakes, L.: Piecewise-quadratics and exponential parameterization for reduced data. Appl. Math. Comput. 221, 620-638 (2013). https://doi.org/10.1016/ j.amc.2013.06.060

13. Kozera, R., Noakes, L.: Piecewise-quadratics and reparameterizations for interpolating reduced data. In: Gerdt, V.P., Koepf, W., Seiler, W.M., Vorozhtsov, E.V. (eds.) CASC 2015. LNCS, vol. 9301, pp. 260-274. Springer, Cham (2015). https:// doi.org/10.1007/978-3-319-24021-3_20

14. Kozera, R., Noakes, L., Wilkołazka, M.: A modified complete spline interpolation and exponential parameterization. In: Saeed, K., Homenda, W. (eds.) CISIM 2015. LNCS, vol. 9339, pp. 98-110. Springer, Cham (2015). https://doi.org/10.1007/9783-319-24369-6_8

15. Kozera, R., Noakes, L., Szmielew, P.: Convergence orders in length estimation with exponential parameterization and $\varepsilon$-uniformly sampled reduced data. Appl. Math. Inf. Sci. 10(1), 107-115 (2016). https://doi.org/10.18576/amis/100110

16. Kuznetsov, E.B., Yakimovich, A.Y.: The best parameterization for parametric interpolation. J. Comput. Appl. Math. 191(2), 239-245 (2006). https://core.ac.uk/download/pdf/81959885.pdf

17. Kvasov, B.I.: Methods of Shape-Preserving Spline Approximation. World Scientific Publishing Company, Singapore (2000). https://doi.org/10.1142/4172

18. Lee, E.T.Y.: Choosing nodes in parametric curve interpolation. Comput. Aided Des. 21(6), 363-370 (1989). https://doi.org/10.1016/0010-4485(89)90003-1

19. Noakes, L., Kozera, R.: Cumulative chords piecewise-quadratics and piecewisecubics. In: Klette, R., Kozera, R., Noakes, L., Weickert, J. (eds.) Geometric Properties of Incomplete Data, Computational Imaging and Vision, chap. 4, vol. 31, pp. 59-75. Springer, Dordrecht (2006). https://doi.org/10.1007/1-4020-3858-8_4

20. Piegl, L., Tiller, W.: The NURBS Book. Springer, Heidelberg (1997). https://doi. org/10.1007/978-3-642-59223-2

21. Rababah, A.: High order approximation methods for curves. Comput. Aided Geom. Des. 12(1), 89-102 (1995). https://doi.org/10.1016/0167-8396(94)00004-C

22. Wolfram, S.: The Mathematica Book. Wolfram Media Inc., Champaign (2003). https://www.wolfram.com/books/profile.cgi?id=4939 